\title{
NOTIFICAÇÕES DA GERÊNCIA DE RISCO E SUA CONTRIBUIÇÃO PARA A SEGURANÇA DO PACIENTE
}

\author{
Anna Priscila Barros de Oliveira ${ }^{1}$, Elizandra Cássia da Silva Oliveira ${ }^{2}$, Regina Célia de Oliveira ${ }^{3}$
}

\begin{abstract}
RESUMO: Objetivou-se identificar as notificações de produtos a tecnovigilância e farmacovigilância a Gerência de risco entre os anos de 2002 a 2004 e de 2007 a 2013. Trata-se de estudo retrospectivo de análise documental com abordagem quantitativa. Os dados foram coletados no período de abril a agosto de 2014, analisados e apresentados em frequência absoluta e relativa. Identificaram-se 529 notificações. A maior frequência de notificação ocorreu nas unidades de internação $n=218$ (42\%). O tipo de notificação Tecnovigilância liderou com $\mathrm{n}=494(93,4 \%)$, o subgrupo venopunção teve prevalência de $\mathrm{n}=21841,2 \%$. O defeito do produto apresentou $\mathrm{n}=32461,2 \%$ das ocorrências notificadas. A associação entre o setor e os materiais notificados destaca-se o grupo de venopunção no setor das Emergências $n=68$ (70,1\%). Estes dados são relevantes para gerar um indicador de qualidade e garantir melhores produtos de saúde que promovam segurança para os pacientes e profissionais de saúde.
\end{abstract}

DESCRITORES: Controle de risco; Garantia da qualidade dos cuidados de saúde; Segurança do paciente; Enfermagem; Gerenciamento de risco.

\section{RISK MANAGEMENT REPORTING AND ITS CONTRIBUTION TO PATIENT SAFETY}

ABSTRACT: The present study aimed to identify risk management reporting of products related to technovigilance and pharmacovigilance between 2002 and 2004 and 2007 and 2013. Retrospective, documentary study with quantitative approach. Data were collected from April to August 2014, analyzed and presented using absolute and relative frequency calculations. A total number of 529 notifications were reported. Most notifications occurred in in-patient units: $\mathrm{n}=218(42 \%)$. The Technovigilance notification type led with $n=494(93.4 \%)$, the subgroup venipuncture had a prevalence of $n=218,41.2 \%$. Defective product corresponded to $\mathrm{n}=324,61.2 \%$ of the reported events. Regarding the association between sector and notified materials, the venipuncture group, in the Emergency department stands out with $n=68(70.1 \%)$. This data contributes to the generation of a quality indicator and to ensure better health products that promote safety to patients and health professionals.

DESCRIPTORS: Risk control; Health care quality assurance; Patient safety; Nursing; Risk management.

\section{NOTIFICACIONES DE LA GESTIÓN DE RIESGO Y SU CONTRIBUCIÓN PARA LA SEGURIDAD DEL PACIENTE}

RESUMEN: Estudio cuyo propósito fue identificar las notificaciones de productos de tecnovigilancia e farmacovigilancia a la Gestión de riesgo entre los años de 2002 a 2004 y de 2007 a 2013. Es un estudio retrospectivo de análisis documental con abordaje cuantitativo. Los datos fueron obtenidos en el periodo de abril a agosto de 2014, analizados y presentados en frecuencia absoluta y relativa. Fueron identificados 529 apuntes. Las unidades de internación tuvieron la mayor frecuencia de notificación: $\mathrm{n}=218$ (42\%). El tipo de apunte tecnovigilancia fue superior, con $n=494$ (93,4\%), el subgrupo venopunción tuvo prevalencia de $n=218$ 41,2\%. El defecto del producto presentó $n=324,61,2 \%$ de las ocurrencias notificadas. La asociación entre el sector y los materiales notificados se destaca en el grupo de venopunción en el sector de las Emergencias $n=68$ (70,1\%). Estos datos son relevantes y pueden generar un indicador de cualidad, además de garantizar mejores productos de salud y promover seguridad a los pacientes y profesionales de salud. DESCRIPTORES: Control de riesgo; Garantía de cualidad de los cuidatos de salud; Seguridad del paciente; Enfermería; Gestión de riesgo.

${ }^{1}$ Enfermeira. Especialista em Suporte Avançada a Vida e Unidade de Tratamento Intensivo. Universidade de Pernambuco. Recife, PE, Brasil.

${ }^{2}$ Enfermeira. Mestre em Enfermagem. Enfermeira Assistencial do Hospital da Restauração em Recife. Recife, PE, Brasil.

${ }^{3}$ Enfermeira. Doutora em Enfermagem. Docente da Faculdade de Enfermagem da Universidade de Pernambuco. Recife, Pernambuco, Brasil.

Autor Correspondente:

Elizandra Cassia da Silva Oliveira

Hospital da Restauração

Estrada do Arraial, 2405 - 52051-380 - Recife, PE, Brasil

Email: elizandra.cassia@bol.com.br
Recebido: 08/06/2016 Finalizado: 07/11/2016 


\section{INTRODUÇÃO}

As mudanças epidemiológicas, demandas demográficas, mudanças político-econômicas e o desenvolvimento de novas soluções tecnológicas para os problemas da saúde, aumentam a complexidade do funcionamento dos serviços em saúde ${ }^{(1)}$. Neste contexto a inserção de novas tecnologias é de suma importância para a recuperação da saúde e a qualidade de vida dos pacientes.

Entretanto, a inovação tecnológica tem gerado problemas como: aceitação indiscriminada e passiva dos países menos desenvolvidos, qualidade insatisfatória, baixa capacitação profissional, uso indevido e custos hospitalares que impactam as organizações hospitalares no Brasil( ${ }^{(2)}$.

$\mathrm{Na}$ busca da melhoria da qualidade dos produtos para a saúde, gerenciamento e monitoração de eventos adversos no ambiente hospitalar a ANVISA (Agência Nacional de Vigilância Sanitária) criou em 2002 a Rede Brasileira de Hospitais Sentinela. A rede conta com 193 hospitais que atuam sistematicamente no monitoramento e notificação de eventos adversos; além de cumprir todos os requisitos de excelência na realização de relatos de problemas para a ANVISA ${ }^{(3-4)}$.

A Rede Sentinela é uma estratégia da Vigilância Sanitária Pós-Uso/Pós-Comercialização de Produtos (Vigipós), que consiste em manter observação sistemática dos eventos adversos e dos desvios de qualidade (queixas técnicas) relacionados aos produtos sob vigilância sanitária. A rede funciona como observatório nos serviços para o gerenciamento de riscos à saúde, e atua em conjunto com o Sistema Nacional de Vigilância Sanitária $(\mathrm{SNVS})^{(5)}$. Cada hospital integrante da Rede Sentinela possui uma Gerencia de Risco (GR), que atua como elemento de ligação com a ANVISA.

A Gerência de Risco desenvolve ações no âmbito da farmacovigilância, que trata do controle e vigilância em medicamentos, da hemovigilância, que aborda informações sobre efeitos colaterais, hemotransfusões e hemocomponentes, da tecnovigilância que consiste na vigilância de insumos e equipamentos hospitalares e biovigilância que destina-se a notificação de reações adversas ocorridas em pessoas doadoras ou receptoras de células, tecidos ou órgãos utilizados em procedimentos de transplantes, enxertos, reprodução humana assistida e/ou terapias avançadas ${ }^{(4-5)}$.

Diante da importância que a GR desempenha no desenvolvimento e manutenção de ações de gerenciamento de risco e segurança do paciente. O presente estudo tem por objetivo identificar as notificações de produtos envolvendo a tecnovigilância e farmacovigilância à Gerência de risco.

\section{METODOLOGIA}

Pesquisa descritiva, retrospectiva, de natureza documental com análise quantitativa dos dados. Realizado no setor de Gerencia de Risco de um Hospital de grande porte com atendimento de alta complexidade em trauma da rede pública estadual do Recife.

Os dados da pesquisa foram coletados da ficha de notificações do serviço da Gerência de Risco durante dez anos de funcionamento. Compreendendo os anos de 2002 a 2004 e de 2007 a 2013. Os anos de 2005 e 2006 foram excluídos por apresentarem descontinuidade no preenchimento das fichas de notificações. O estudo totalizou 529 notificações. As fichas de notificação continham as seguintes variáveis: ano, grupo de materiais, tipo de notificação, local de ocorrência (setor) e grupo de ocorrências.

Em virtude da grande variedade do grupo de materiais notificados; estes foram separados em grupos: 1. Materiais de venopunção que contemplou as notificações relacionadas as seringas, cateteres periféricos, dispositivos de conexão de duplo e triplo lúmen, esparadrapo, soros e equipos de infusão venosa; 2 . Sondas, drenos e cânulas ficaram em um grupo particular, composto por cânulas endotraqueais, drenos torácicos, sondas gástricas, uretrais, retais e de aspiração; 3 . Materiais cirúrgicos que inclui as luvas, compressas, gazes, ataduras, fios de sutura, pinças, agulhas, lâminas de bisturi, toucas e máscaras; 4. Equipamentos e Material médico hospitalar incluindo aspiradores, colares cervicais, pinceta, esfigmanômetro, bolsa de colostomia, bomba de infusão, monitores cardíacos, respiradores e detergentes enzimáticos; 5 . Fármacos foram formados por todas as queixas registradas de medicamentos. 
A coleta de dados foi realizada de 01 de abril a 30 de agosto de 2014 após aprovação pelo Comitê de Ética em Pesquisa do Complexo Hospitalar (HUOC/PROCAPE) conforme número de aprovação do parecer 11555912.8.0000.5192.

Após coletados, os dados foram organizados e analisados por distribuições absolutas e percentuais. O programa estatístico utilizado para a digitação dos dados e obtenção dos cálculos estatísticos foi o Statistical Package for the Social Sciences (SPSS) na versão 21. Para verificar a associação entre duas variáveis categóricas foi utilizado o teste Qui-quadrado de Pearson e/ou teste da Razão de Verossimilhança. A margem de erro utilizada nas decisões dos testes estatísticos foi de 5\%.

\section{RESULTADOS}

A análise deste estudo, realizado no período de 10 anos, apontou 529 notificações que encontramse disposta em gráficos e tabelas. Na Figura 1, verificou-se que o ano com maior frequência foi 2010 (23,4\%), seguido dos anos 2009 (17,6\%), 2003 (12,5\%) e 2011 (12,5\%) e os outros anos tiveram percentuais que variaram de 3 a $8,6 \%$.

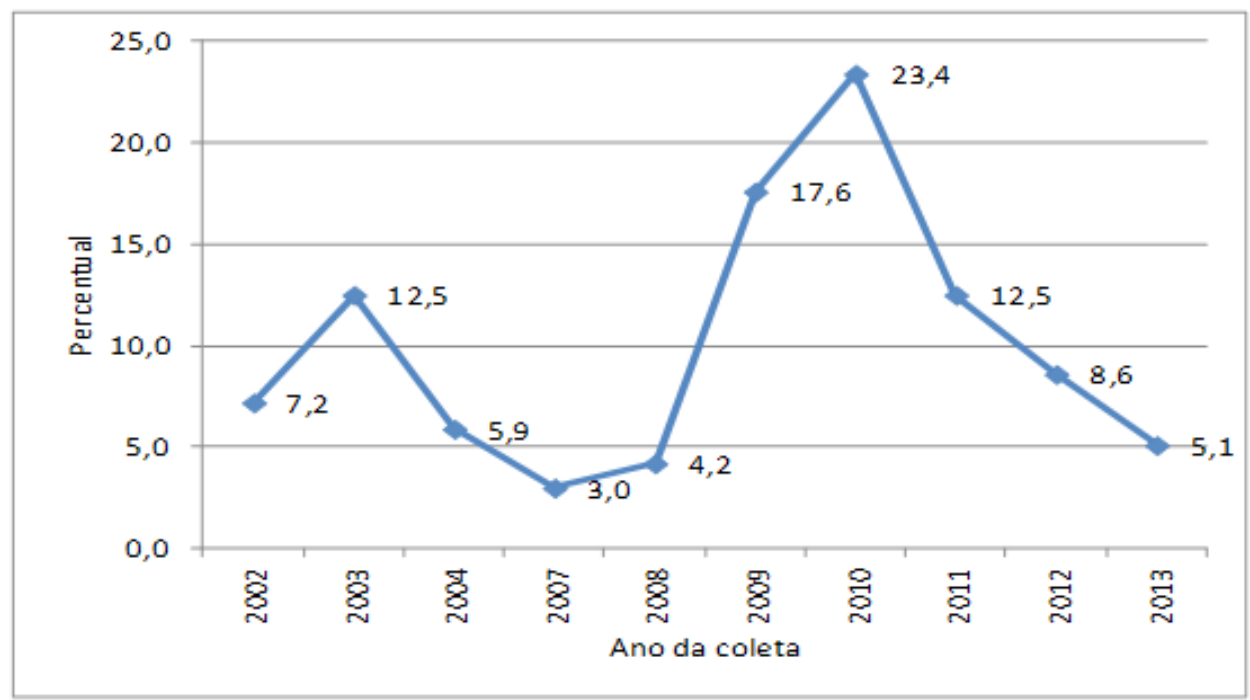

Figura 1 - Distribuição absoluta das notificações da Gerencia de Risco no período 2002 a 2013. Recife, PE, Brasil, 2013

A Tabela 1 caracteriza as notificações realizadas a Gerência de Risco segundo as variáveis analisadas no estudo.

Tabela 1 - Caracterização das Notificações a Gerência de Risco segundo as variáveis: grupo de material, tipo de notificação, setor e tipo de ocorrência (N=529). Recife, PE, Brasil, 2013 (continua)

\begin{tabular}{lcc} 
Variável & $\mathbf{N}$ & $\mathbf{0}$ \\
& $\mathbf{5 2 9}$ & $\mathbf{1 0 0}$ \\
\hline Grupo de material & & \\
\hline Venopunção & 218 & 41,2 \\
\hline Material cirúrgico & 173 & 32,7 \\
\hline Equipamentos e Material médico hospitalar & 78 & 14,7 \\
\hline Sondas/dreno e cânulas & 21 & 4 \\
\hline Fármacos & 33 & 6,2 \\
\hline Notificação Incompleta & 6 & 1,1 \\
\hline Tipo de notificação & & \\
\hline Tecnovigilância & 494 & 93,4 \\
\hline Farmacovigilância & 30 & 5,7 \\
\hline
\end{tabular}




\begin{tabular}{lcc}
\hline Saneante & 4 & 0,8 \\
\hline Notificação Incompleta & 1 & 0,2 \\
\hline Setor & & \\
\hline Clínicas Diversas & 224 & 42,3 \\
\hline Bloco cirúrgico & 134 & 25,3 \\
\hline Emergências & 98 & 18,5 \\
\hline UTIs & 67 & 12,7 \\
\hline Notificação Incompleta & 6 & 1,1 \\
\hline Tipo de Ocorrência & & \\
\hline Defeito & 324 & 61,2 \\
\hline Vazamento & 64 & 12,1 \\
\hline Embalagem & 49 & 9,3 \\
\hline Resíduo/ sujidade/ corpo estranho & 34 & 6,4 \\
\hline Notificação Incompleta & 22 & 4,2 \\
\hline Coloração, consistência e odor alterado & 20 & 3,8 \\
\hline Evento/ efeito adverso/sem efeito & 13 & 2,5 \\
\hline Validade & 3 & 0,6
\end{tabular}

Quanto ao grupo de material o grupo de materiais Venopunção foi o material mais utilizado com $\mathrm{n}=218$ 41,2\%, seguido do Material cirúrgico $\mathrm{n}=173$ (32,7\%) e Equipamentos e Material médico hospitalar $\mathrm{n}=78(14,7 \%)$. Tratando-se da área de notificação verificou-se que a tecnovigilancia obteve percentuais de queixas com n=494 (93,4\%), Farmacovigilância e Saneante tiveram percentuais com n=30 (5,7\%) e quatro $(0,8 \%)$ respectivamente.

Em relação aos setores o maior percentual (42,3\%) foi procedente dos andares, seguidos dos procedentes do bloco cirúrgico $(25,3 \%)$ e emergências $(18,5 \%)$ e com menor percentual foi registrado os procedentes das UTI's (12,7\%). Quanto ao tipo de ocorrência n=324 (61,2\%) foi referido a Defeito e Vazamento, Embalagem e Resíduo/ sujidade/ corpo estranho tiveram percentuais respectivos de $\mathrm{n}=64$ $(12,1 \%), n=49(9,3 \%)$ em=34( $6,4 \%)$.

Na Tabela 2 observa-se a associação entre o setor e os materiais notificados, onde se destaca as maiores diferenças: O grupo de venopunção no setor das Emergências (70,1\%) e no Bloco Cirúrgico de $(20,9 \%)$. O grupo de material cirúrgico no Bloco Cirúrgico (68,7\%). O grupo de material de apoio e mobília nas UTIs (25,8\%). A associação entre as duas variáveis se mostra significativa $(p<0,001)$.

Tabela 2 - Distribuição do Grupo de Materiais Notificados à Gerência de Risco segundo os Setores (N=517). Recife, PE, Brasil, 2013

\begin{tabular}{|c|c|c|c|c|c|c|c|c|c|c|c|}
\hline \multirow[t]{2}{*}{ Grupo Material } & \multicolumn{2}{|c|}{$\begin{array}{c}\text { Bloco } \\
\text { cirúrgico }\end{array}$} & \multicolumn{2}{|c|}{ Emergências } & \multicolumn{2}{|c|}{$\begin{array}{l}\text { Clínicas } \\
\text { Diversas }\end{array}$} & \multicolumn{2}{|c|}{ UTIs } & \multicolumn{2}{|c|}{ Grupo total } & \multirow[t]{2}{*}{$\begin{array}{l}\text { Valor } \\
\text { de p }\end{array}$} \\
\hline & $\mathbf{n}$ & $\%$ & $\mathbf{N}$ & $\%$ & $\mathbf{n}$ & $\%$ & $\mathbf{n}$ & $\%$ & $\mathbf{N}$ & $\%$ & \\
\hline Venopunção & 28 & 20,9 & 68 & 70,1 & 93 & 42,3 & 27 & 40,9 & 216 & 41,8 & $\begin{array}{l}\mathrm{p}(1)< \\
0,001^{*}\end{array}$ \\
\hline Sondas/dreno e cânulas & 4 & 3 & 5 & 5,2 & 6 & 2,7 & 6 & 9,1 & 21 & 4,1 & \\
\hline Material cirúrgico & 92 & 68,7 & 14 & 14,4 & 55 & 25 & 10 & 15,2 & 171 & 33,1 & \\
\hline $\begin{array}{l}\text { Equipamentos e Material } \\
\text { médico hospitalar }\end{array}$ & 6 & 4,5 & 8 & 8,2 & 47 & 21,4 & 17 & 25,8 & 78 & 15,1 & \\
\hline Fármacos & 4 & 3 & 2 & 2,1 & 19 & 8,6 & 6 & 9,1 & 31 & 6 & \\
\hline TOTAL & 134 & 100 & 97 & 100 & 220 & 100 & 66 & 100 & 517 & 100 & \\
\hline
\end{tabular}

(*): Diferença significativa a $5 \%$.

(1): Através do teste da Razão de Verossimilhança.

Obs: houve 6 notificações incompletas referente a grupo de material e 6 notificações incompletas referente a setores. 
Os resultados deste estudo mostraram um crescimento considerável do número de incidentes notificados entre os períodos de 2002 a 2008 e de 2009 a 2013, saltando de 170 (32,5\%) para 353 (67,4\%), respectivamente. Fazendo uma breve associação com alguns dos dados nacionais registrados no NOTIVISA (Notificações para a Vigilância Sanitária) observa-se um aumento gradual do total de notificações. Em 2006 são registrados 102 queixas técnicas, 2010 com 26.997 queixas técnicas registradas e em 2013 há 38.839 queixas registrada(6). Tal fato pode ser destacado a partir do crescimento da implantação de gerências de risco, partindo da rede de hospitais sentinela pelo país, motivado pela disseminação da campanha de divulgação das seis metas internacionais de qualidade e segurança do paciente, preconizadas pela Organização Mundial de Saúde, no ano de 2010. No entanto, apesar do aumento no número total de notificações ano a ano; observa-se um número pouco expressivo de incidentes notificados se associarmos a complexidade do cuidado prestado no hospital campo de pesquisa.

Vê-se que a queixa técnica é uma notificação feita pelo profissional de saúde, quando observado um desvio dos parâmetros de qualidade exigidos para a comercialização ou aprovação no processo de registro de um produto farmacêutico, ou seja, quando se suspeita de qualquer desvio de qualidade ${ }^{(7)}$. A notificação de queixas técnicas e eventos adversos contribuem para o controle e prevenção das ocorrências associadas aos produtos de saúde e consequentemente a melhoria da qualidade destes para a utilização na população em geral.

O aumento no número de notificações está diretamente relacionado a ações educativas realizadas com os profissionais, a qual se faz necessário o empenho da GR em realizar atividades de incentivo a notificações ${ }^{(8-9)}$.

Entretanto, estudos nacionais e internacionais apontam para a presença de subnotificação ou a não notificação associada às notificações voluntárias que constituem uma forma simples e participativa de notificar as queixas pelos profissionais ${ }^{(10-11)}$. Dentre os principais desafios a serem superados pela subnotificação temos a escassez e sobrecarga de pessoal nos estabelecimento de saúde, o tempo gasto com o preenchimento da ficha, a falta de compreensão dos incidentes e a ausência de retorno quanto à providência tomada ${ }^{(12-13)}$.

Outro ponto importante para a subnotificação está associado aos profissionais que trabalham na GR (Gerência de Risco) que necessitam de treinamento permanente a fim de se evitar falhas na realização da busca ativa, e no preenchimento correto da ficha de notificação, o que acaba comprometendo o banco de dados da unidade, dificultando dessa forma a contabilidade dos casos e dificultando eventuais pesquisas ${ }^{(14)}$.

As notificações manuais caracterizam barreiras para adequação do processo de notificação. Estudo mostrou que $26,1 \%$ das notificações manuais apresentavam erros na escolha da ficha para notificação ou erros na marcação do incidente, 49,6\% não tinha a gravidade do problema, além disso, 36,8\% dessas notificações manuscritas apresentaram problemas quanto à legibilidade, sendo consideradas ilegíveis e $22,3 \%$ delas estavam rasuradas ${ }^{(10)}$.

No que diz respeito à identificação das notificações enviadas à Gerência de Risco observa-se que aquelas relacionadas à Tecnovigilância lideraram com $n=494(93,4 \%)$, sendo as Clínicas diversas o maior notificador com $n=224$ (42\%) seguido do setor Bloco Cirúrgico com n=134 (25\%) do total de queixas registradas.

A partir do ano de 2009, a ferramenta para monitorar as notificações passou a ser a NOTIVISA. Os relatos de notificações de tecnovigilância não eram compulsórios até o ano de 2010, quando foi criada a Resolução da Diretoria Colegiada - RDC 67/2009(15). A consciência da importância de exercer a atividade de notificar está sendo lentamente desenvolvida no Brasil.

Assim, ao longo dos anos os artigos médico-hospitalares obtiveram crescente número de queixas no país com 5.416 queixas em 2009, 7.070 queixas em 2010, 8.994 queixas em 2011 e 10.225 queixas em 2012, enquanto que no estudo aplicado, 2010 foi o ano que mais obteve queixas $(23,4 \%)$, seguido do ano de 2009 (17,6\%), mantendo assim o mesmo crescimento cronológico das notificações nacionais 
registradas pelo NOTIVISA ${ }^{(6)}$.

Um dado importante a ser avaliado quanto a um número maior de notificações na área de Tecnovigilância, pode está possivelmente relacionada ao não envolvimento de algum tipo de erro do profissional, serem facilmente aceitas e notificadas pelos profissionais ${ }^{(16)}$.

O presente estudo revelou que as Clínicas Diversas foi o setor de maior notificação. Resultado este, provavelmente relacionado a um maior número de leitos e pacientes internados.

Entretanto, destacamos o setor Bloco Cirúrgico por despertar nas equipes médicas e de enfermagem uma maior participação na prática do gerenciamento de risco. Estas equipes passam a agregar novas referências à avaliação do tratamento a ser prescrito e do material a ser utilizado ${ }^{(17)}$. Os enfermeiros aprendem a relacionar eventos da prática de cuidados com os possíveis riscos decorrentes do uso de materiais. Reforçado também pela implementação do protocolo da cirurgia segura, que exige a verificação do funcionamento do monitor multiparamétrico antes da indução anestésica e da incisão cirúrgica e se as condições dos equipamentos foram avaliadas pela equipe de enfermagem, contribuindo assim para maiores números de notificações ${ }^{(18)}$.

Quanto ao grupo de materiais observamos que o grupo de venopunção representou $41,2 \%$ do total das queixas técnicas. Caracterizando o mesmo perfil encontrado em nosso estudo; um estudo realizado com 393 queixas técnicas de produtos a ANVISA revelou que 53,4\% das notificações eram relacionadas à Equipos (18,9\%), Seringas Descartáveis (11,8\%), Cateteres (8,3\%), Luvas Descartáveis $(6,4 \%)$, Luvas Cirúrgicas $(4,2 \%)$, Sondas $(3,8 \%)^{(6)}$.

Vê-se que este grupo de materiais é relevante na realização de procedimentos diagnósticos, cirúrgicos e tratamentos clínicos com alto risco para eventos adversos. Apesar de serem produtos classificados de Médio Risco (Classe II) e relativamente baratos, são produtos amplamente utilizados; merecem maior atenção do ponto de vista da vigilância sanitária, principalmente na fase da póscomercialização ${ }^{(6)}$.

Entretanto, o número de queixas com farmacoviligilantes no referido hospital, levando-se em consideração o seu porte e sua representação estadual, foi bem abaixo do esperado. O perfil das notificações referentes a produtos/motivos, disponibilizadas pelo NOTIVISA no período de 2006 a 2011 foi liderada pelo grupo medicamentos nos anos 2008 com 5.707,2009 com 7.602, 2010 com 9.586 e 2011 com 12.573 notificações ${ }^{(19)}$. Enquanto que em nosso estudo foi visto o registro de nove queixas de 2002 a 2008 e 24 queixas de 2009 a 2013, emergindo assim, a necessidade de intervenções educativas, como estratégia de sensibilização do profissional para o ato de notificar.

Neste contexto é importante que o profissional da saúde saiba reconhecer uma reação adversa a medicamentos (RAM). RAM é uma resposta a um medicamento, que é nociva e não-intencional e que ocorre nas doses normalmente usadas em seres humanos. Visando a prevenção e segurança dos tratamentos farmacológicos como também os riscos relativos à exposição a eles. A detecção de reações adversas, muitas vezes, não são reveladas nos ensaios clínicos pré-comercialização, e provavelmente, reduz as taxas de morbidade e mortalidade por uso de medicamentos. Quando detectado qualquer evento adverso, o profissional de saúde deve notificá- ${ }^{\left({ }^{(20)}\right.}$. Quando não há notificação, perdemos a oportunidade de novos estudos de medicamentos relacionados a risco e benefícios, interações medicamentosas, via de administração, qualidade de fabricação, entre outros ${ }^{(21)}$.

Quanto ao tipo de ocorrência obtivemos defeito do produto com n=324 61,2\% das notificações relacionado à sua estrutura. Relatório da ANVISA revela que a classificação da notificação pelo modo de falha (não funciona) apontou que o produto não atende os requisitos de efetividade previstos em normativo regulatório com 2.906 notificações ${ }^{(6)}$. Alerta a estes produtos são essenciais para monitorar a qualidade no processo industrial e, consequentemente, garantir segurança ao paciente.

Outro achado desta investigação foi à notificação do grupo de venopunção com n=68 (70,1\%) das queixas técnicas no setor das Emergências. Tradicionalmente é um setor onde o uso da punção venosa é considerado vital para o desenvolvimento das atividades, o que proporciona maiores habilidades de observação e avaliação desses itens. O processo de cuidar nas unidades especializadas depende em grande parte do uso seguro de materiais e equipamentos de suporte à vida, entre outros procedimentos, que também requerem políticas eficientes de qualidade e manutenção efetiva ${ }^{(12)}$. 
Os saneantes obtiveram apenas quatro queixas, confirmando ser o grupo de menor notificação, uma vez que os dados nacionais referiram 48 notificações em 2007, 91 em 2008, 140 em 2009, 215 em 2010, 120 em 2011 e 159 em 2012 ${ }^{(17)}$. Dentre as queixas encontradas com saneantes está o odor forte, pouca espuma e diluição acima do esperado.

\section{- CONCLUSÃO}

As queixas técnicas relacionadas a tecnovigilância, o grupo de materiais de venopunção e o tipo de ocorrência defeito dos materiais médico-hospitalares caracterizaram as notificações do estudo. Estes dados são relevantes para gerar indicador de qualidade, e contribuir no monitoramento de melhores produtos de saúde no mercado. Promovendo segurança para os pacientes e profissionais de saúde.

O monitoramento dos produtos na etapa de pós-comercialização, por meio das queixas técnicas deve ser contínuo, de maneira a identificar precocemente as necessidades de intervenção, bem como a sistematização das ações e condutas a serem adotadas.

Dentro deste contexto a estratégia do hospital sentinela exerceu seu objetivo e efetivação de suas ações ao que tange a vigilância em saúde de produtos comercializados.

Este estudo apresentou limitações quanto ao registro das notificações incompletas. Sugerimos implantação de notificações informatizada e sistematizada para uma gestão eficiente; bem como conscientização no cotidianos dos profissionais da área da saúde que a efetivação do gerenciamento de risco é responsabilidade de todos e não somente dos gerentes.

\section{REFERÊNCIAS}

1. Ministério da Saúde (BR). Secretaria de Ciência, Tecnologia e Insumos Estratégicos. Departamento de Ciência e Tecnologia. Diretrizes metodológicas: elaboração de estudos para avaliação de equipamentos médicos assistenciais. Brasília: Ministério da Saúde. 2013.

2. Kuwabara CCT, Évora YDM, de Oliveira MMB. Gerenciamento de risco em tecnovigilância: construção evalidação de instrumento de avaliação de produto médico-hospitalar. Rev. Latino-Am. Enfermagem. 2010;18(5):943-51.

3. Brasil. Portaria n. 1.693, de 8 de novembro de 2011. Dispõe sobre Critérios para Credenciamento de Instituições na Rede Sentinela - SUS. Diário Oficial da União, n. 215, Brasília, 2011.

4. Ministério da Saúde (BR). Agência Nacional de Vigilância Sanitária. Rede Sentinela. [acesso em 15 jun 2015]. Disponível: http://www.anvisa.gov.br/servicosaude/hsentinela/historico.htm.

5. Agência Nacional de Vigilância Sanitária (ANVISA). Blog da Rede Sentinela. Histórico da rede. [Internet] Brasília (DF): Agência Nacional de Vigilância Sanitária; 2015 [acesso 16 mai 2016]. Disponível: http://redesentinela-anvisa. blogspot.com.br/p/historico-da-rede.html.

6. Vicente MG, Freitas DRC. Comportamento dos materiais médico-hospitalares no Brasil a partir dos dados de notificação em Tecnovigilância. Boletim Informativo de Tecnovigilância. [Internet] Brasília; 2012 [acesso em 10 jun 2016]. Disponível: http://www.anvisa.gov.br/boletim_tecno/boletim_tecno_agosto_2012/PDF/3BIT-4-2012Comportamento\%20Materiais\%20M\%C3\%A9dico-hospitalares.pdf.

7. Agência Nacional de Vigilância Sanitária (ANVISA). Manual de Tecnovigilância: abordagens de vigilância sanitária de produtos para a saúde comercializados no Brasil. Brasília (DF): Ministério da Saúde; 2010.

8. Pereira Primo L, Capucho HC. Intervenções educativas para estímulo a notificações voluntárias em um hospital de ensino da rede sentinela. R. Bras. Farm. Hosp. Serv. Saúde. 2011;2(2):26-30.

9. Costa VT, Meirelles BHS, Erdmann AL. Best practice of nurse managers in risk management. Rev. Latino-Am. Enfermagem. 2013;21(5):1165-71.

10. Capucho HC, Arnas ER, Cassiani SHB. Segurança do paciente: comparação entre notificações voluntárias manuscritas e informatizadas sobre incidentes em saúde. Rev. Gaúcha Enferm. 2013;34(1):164-72. 
11. Lorenzini E, Santi JAR, Báo ACP. Segurança do paciente: análise dos incidentes notificados em um hospital do sul do Brasil. Rev. Gaúcha Enferm. 2014;35(2):121-7.

12. Fernandes AMML, Queiroz PJP. Cultura de Segurança do Doente percepcionada por enfermeiros em hospitais distritais portugueses. Rev. Enf. Ref. 2011;3(4):37-48.

13. Gil RB, Chaves LDP, Laus AM. Gerenciamento de recursos materiais com enfoque na queixa técnica. Rev. Eletr. Enf. 2015;17(1):100-7.

14. Ministério da saúde (BR). Agência nacional de vigilância sanitária. Experiências da Rede Sentinela para a Vigilância Sanitária. Uma referência para o Programa Nacional de Segurança do Paciente. Brasília: Ministério da Saúde; 2013.

15. Brasil. Agencia Nacional de Vigilância Sanitária - ANVISA. Resolução RDC no 67 de 21 de dezembro de 2009. Dispõe sobre normas de tecnovigilância aplicáveis aos detentores de registro de produtos para saúde no Brasil. Diário Oficial da República Federativa do Brasil, Brasília, dez. 2009.

16. da Silva RFA, Barreiro Filho RD, Santos M, Nascimento MAL. Estratégia educacional como contribuinte ao gerenciamento de risco hospitalar: estudo quase experimental. Online braz. j. nurs. (Online). 2001;10(1):1-8.

17. Bohomol E, Tartali JA. Eventos adversos em pacientes cirúrgicos: conhecimento dos profissionais de enfermagem. Acta paul. enferm. 2013;26(4):376-81.

18. Ministério da Saúde (BR). Agência Nacional de Vigilância Sanitária. Organização Mundial da Saúde. Segundo desafio global para a segurança do paciente. Cirurgias seguras salvam vidas. Rio de Janeiro: Organização PanAmericana da Saúde; 2009.

19. de Oliveira JR, Xavier RMF, Santos Junior AF. Eventos adversos notificados ao Sistema Nacional de Notificações para a Vigilância Sanitária (NOTIVISA): Brasil, estudo descritivo no período 2006 a 2011. Epidemiol. Serv. Saúde. 2013;22(4):671-8.

20. de Menezes FG, Nascimento JWL. Monitoramento de eventos adversos em ambiente hospitalar: relato de farmacovigilância, São Paulo. ConScientiae Saúde. 2010;9(4):582-7.

21. Lopez AV. La farmacovigilancia y la tecnovigilancia em mi práctica diaria. Diagnostico. 2014;53(1):51-4. 